\title{
Barwise and Etchemendy's Theory of Truth
}

\section{HIROSHI AOYAMA}

Tokai Industrial College

Barwise and Etchemendy, whom we will call 'B\&E' in what follows, have recently presented a novel solution to the Liar paradox in their very attractive book The Liar: An Essay in Truth and Circularity. ${ }^{1}$ Their solution is given in the theory of truth which they have developed using Austin's account of truth. ${ }^{2}$ Their theory of truth is well developed both philosophically and technically. In this paper, we will first look at the philosophical aspect of their theory of truth and then give some criticisms of it.

\section{Philosophical Aspect of B\&E's Theory of Truth}

\subsection{The bearers of truth}

Before discussing their solution to the Llar paradox, they make decisions on certain important matters related to the paradox. The most important decision is on what are the primary bearers of truth. B\&E say that propositions are the primary bearers of truth. What a sentence like "I hit the policeman" expresses is highly dependent on the contexts or situations in which it is used. One does not know whether or not what it expresses is true at least until he knows such contexts or situations. Therefore, assigning truth values to sentences does not make sense. They should be assigned to what the sentences express in the contexts in which they are used, i.e., to the propositions they express. B\&E then consider the distinction between statements and propositions. They say,

By a statement we will understand certain sorts of datable events, those where a speaker asserts or attempts to assert something using a declarative sentence. In contrast, we take a proposition to

1This is a revised version of a chapter of my Ph.D. thesis, This is the Title of this Thesis, submitted to the University of Illinois at Urbana-Champaign in May, 1988. I am very grateful to my thesis adviser, Professor Timothy McCarthy, for his invaluable help. I am also very grateful to Professor Jon Barwise. Before the publication of Barwise and Etchemendy (1987), he sent me a draft of the book. He also gave me some important comments on the first draft of that chapter of my thesis.

It appears in Austin (1950). Austin, John L. "Truth." Proceedings of the Aristotelian Society. supp. vol. xxiv. 
be a claim about the world, the kind of thing that is asserted by a successful statement. ${ }^{3}$

The two statements "I am tired" uttered by me and "Hiroshi is tired" uttered by a friend of mine express the same proposition. Statements may fail to have truth values because of the failure of the presuppositions they involve but propositions cannot because they are the claims made by statements whose presuppositions are fulfilled. Thus, for example, the sentence "The present king of France is crazy" uttered by me now is a statement but cannot express a proposition because the present king of France does not exist now. Then B\&E make a very important decision on propositions. They say that there are only two possibilities about a proposition; that is, it is either true or not true and its being nof true is just its being false. So, the principle of bivalence holds for propositions, but not for sentences or statements.

Because they take propositions to be the primary bearers of truth, they discard the sentential version of the Liar sentence "This sentence is not true:" They instead use the propositional version of it "This proposition is not true" throughout their book where the phrase This proposition' is intended to refer to the proposition, if any, expressed by the whole sentence containing it. However, the question here is whether or not there really exists any proposition expressed by the Liar sentence. B\&E simply assume, with no real explanation, that the Llar sentence does express a proposition, which they call the 'Liar proposition.' Moreover, they think that there is nothing wrong with sentences which refer to the very propositions they express. Thus, as with the Liar sentence, the phrase This proposition' in the Truth-teller sentence "This proposition is true" refers to the proposition expressed by the Truth-teller sentence itself. Similarly, for the phrase 'this proposition' in the sentence "Max believes this proposition."

\subsection{Modelling propositions}

B\&E present a formal language $L$ in which Liar-like sentences can be expressed and then present its semantics. The Liar-like sentences are interpreted as propositions in the semantics. Although we do not go into the technical side of their truth theory, we mention one technical point: their semantics is based not on the ordinary ZFC set theory but on a new set theory, which they call, ZFC/AFA. B\&E want propositions to be

${ }^{3}$ Barwise, J. and Etchemendy J. The Liar: An Essay in Truth and Circularity. Oxford: Oxford University Press, 1987. p.11.

"ZFC" stands for the Zermelo-Fraenkel set theory with the Axiom of Choice. It has several axioms like the Axiom of Power Set, the Axiom of 
modelled as sets in the universe of ZFC/AFA and the Liar proposition to be modelled as a set containing itself as a member. They need a set theory different from ZFC in which sets containing themselves as members cannot be expressed. ZFC/AFA does the required job right.

To model propositions in ZFC/AFA, B\&E first use Russell's account of truth. However, they then abandon the Russellian propositions for certain reasons and take up Austin's account of truth. They explain it as follows:

a legitimate statement A provides two things: a historical (or actual) situation $S_{A}$, and a type of situation $T_{A}$. The former is just some limited portion of the real world; the speaker refers to it using what Austin calls "demonstrative conventions." The latter is, roughly speaking, a property of situations determined from the statement by means of "descriptive conventions" associated with the language. The statement $A$ is true if $S_{A}$ is of type $T_{A}$; otherwise it is false. 5

B\&E's idea of truth basically agrees with Austin's account of truth. As can be seen from this quotation, Austin takes statements to be the bearers of truth. However, since B\&E take propositions to be the bearers of truth, they say that the proposition p expressed by a statement $A$ is true if the historical situation $p$ is about (About(p)) is of the type of situation (Type(p)) associated with the statement $A$ by the descriptive conventions. They also regard a proposition $p$ as the claim that About(p) is of type Type(p), which they write $p=(A b o u t(p) ;$ Type(p)). Concerning the demonstrative conventions, they say, "The demonstrative conventions require that a statement be about a situation, and situations are portions of the world." On the other hand, they say, "The descriptive conventions give us types of

Replacement, and so on. For the detail of this set theory, consult any standard textbook of set theory. 'AFA' stands for (Aczel's) Anti-Foundation Axiom. In order to state AFA, we need a few definitions. The following are from B\&E (1987), Pp. 39-40, Ch. 3. A graph is a set of nodes and directed edges. If there is an edge $x \rightarrow y$ from node $x$ to node $y$, then $y$ is said to be a child of $x$. A node with no arrow starting from it is said to be childless. A tagged graph is a graph in which each childless node $x$ is tagged by an object tag $(x)$, which is either an atom (unrelement) or the empty set. A decoration for a tagged graph is a function $D$ defined on the nodes of the graph such that for each node $x$, if $x$ has no children, then $D(x)=\operatorname{tag}(x)$; otherwise $D(x)=D(y): y$ is a child of $x$. Given these definitions, we can state AFA. It asserts that every tagged graph has a unique decoration.

5B\&E, (1987), pp. 28-29, Ch. 2.

${ }^{6} B \& E$, (1987), p. 30, Ch. 2. 
situations, and these types are themselves much like Russellian propositions."7 A Russellian proposition, in the simplest case, consists of two things: one object and one property, and it makes the claim that the object has the property. For example, a statement made with the sentence "Tom has a cat" will express a proposition claiming that Tom has the property having a cat.

As for a solution to the Llar paradox, B\&E claim that the Liar proposition can be elther true in a situation or false in another; that is, its truth depends upon situations. This somewhat strange claim is a result of their formal modelling of Austinian propositions. They, however, then introduce the notion of an actual situation and claim that the Liar proposition is false in an actual situation; this is their solution to the Liar paradox.8 The technical formulation of this solution seems to prevent it from yielding contradictions.

In the next section, we will discuss not their solutton to the paradox but rather some of the hypotheses behind it.

\section{Some Discussions of B\&E's Theory of Truth}

\section{Propositions as the primary bearers of truth}

We agree with B\&E that it does not make sense to assign truth values to sentences. The possible truth value of the sentence "I hit the policeman" depends on who 'I' and 'the policeman' refer to. In order to determine its truth value we have to know the situation or context that the sentence is about. Such a situation determines what the sentence expresses, l.e., the proposition it expresses. Thus it is more reasonable to assign truth values to the propositions sentences express. We also think that B\&E's distinction between a proposition and a statement is reasonable. We then agree with them that statements can fail to express propositions and that they fail to have truth values unless they express propositions. Thus we think, as B\&E do, that propositions are the primary

\footnotetext{
7B\&E, (1987), p. 30, Ch. 2

Technically speaking, a situation is a set of states of affairs which they also call 'facts' and which can be written as, e.g., $\langle\mathrm{H}, \mathrm{Claire,} A Y ; 1>$ and $\langle\mathrm{H}$, Claire, AV ; $0>$; the former corresponds to the fact that Claire has the ace of hearts and the latter to the fact that Claire does not have it. A situation $s$ is actual in a model $M$ of the world if 8 is a subset of $M$, where a model of the world is a collection of states of affairs. For more, see B\&E (1987), p. 131, Ch.9.
} 
bearers of truth. 9 Statements and sentences may be said to have truth values in a derivative way, i.e., if they can express propositions; or they may be said to inherit their truth values, if any, from the propositions they express. We also agree with them that every proposition is either true or false (i.e., not true); that is, the principle of bivalence holds for propositions. ${ }^{10}$

In what follows, we take as the Liar sentence "This proposition is false" instead of "This sentence is false."11

\section{On circular (or, self-referential) propositions}

It is certainly true that the term 'this' in a sentence can refer to the very sentence. Sentences like "This is an English sentence" and "This is not italicized" are perfectly understandable sentences when the demonstrative 'This' is taken to refer to the whole sentence in which it occurs. Sentences are physically expressed, orally or visually. As such, it is easy to identify them. However, if the term 'this' is used to refer to the proposition, if any, expressed by a sentence or statement in which it occurs, the identification of the proposition is sometimes very difficult or even impossible. Suppose now that 1 uttered the following sentence:

\section{I know this,}

where the demonstrative 'this' is intended to refer to the proposition, if any, expressed by the sentence (1); that is, (1) may be rewritten as "I know the proposition expressed by this very sentence." Now, the question is whether or not (1) expresses a proposition. Let us check to see if (1) expresses a proposition. The phrase 'I know' is just an indicator of one's knowledge. ${ }^{12}$ As such, it does not express any proposition until some proposition or some phrase referring to a proposition is attached to it.

9In other words, truth (and falsity) is a property of propositions. Truth values here mean True and False, not including a third truth value like Undefined.

${ }^{10}$ Someone might claim that some propositions can be neither true nor false. To examine such a claim, we need to consider various sentences and the propositions they express. We, however, will not do 80 in this paper.

"We also regard "This proposition is not true" as (a version of) the Liar sentence. However, we do not regard "This statement is false" or "This statement is not true" as the Liar sentence since statements as well as sentences are not the primary bearers of truth.

${ }^{12}$ Although there are other usages of the phrase 'I know;' we consider only this usage here. 
express a proposition only when the demonstrative 'this' in it successfully refers to a proposition. But we have been assuming that it refers to the proposition, if any, expressed by (1). So, we have again to check to see if (1) expresses a proposition. This process of checking will go on forever without reaching an end; it is a vicious circle. The upshot of this is that (1) does not express a proposition. ${ }^{13}$

There seem to be many other sentences similar to (1) which do not express propositions. We mention a few: "I believe this," "I understand this," "I hope this," "I can prove this," "I forgot this," and "I corrected this."14

The next question is whether or not there is any proposition like the Liar proposition expressed by the Liar sentence "This proposition is false." We think that there is no such proposition. Let us now check to see if the Llar sentence expresses a proposition. The phrase 'is false' in the sentence has nothing but a function of ascribing falsity to the proposition to which the phrase is attached. ${ }^{15}$ Thus, it cannot express any proposition until it is attached to a proposition or a phrase referring to a proposition. In the case of the Llar sentence, it is attached to the phrase This proposition.' So, we have to ask which proposition this 'This proposition' refers to. But this is tantamount to asking what proposition, if any, the Liar sentence expresses, because it has been assumed that 'This proposition' refers to the proposition, if any, expressed by the Liar sentence. To answer the latter question, we again have to ask if the Liar sentence expresses any proposition at all. Thus we have come back to the question we started with. A vicious circle, again. The upshot of this is of course that the Liar sentence does not express any proposition. ${ }^{16}$

If we had chosen "This sentence is false" as the Liar sentence, we could have easily answered the question "Which sentence does the phrase "This sentence' in the Liar sentence refer to?" The answer would have been "The Liar sentence itself." This is 80 because sentences, or tokens of

${ }^{13}$ An analogous observation is made in Ryle (1951). Ryle, Gilbert. "Meterologicality." Analysis: Vol. 11 (1951): 61-69. He uses, what he calls, the 'namely-rider.'

11'In each of these examples, the term 'this' is intended to refer to the proposition, if any, expressed by the whole sentence in which it appears. The reader is asked to check these sentences to see how absurd they are. In the last sentence, the past tense of the verb seems to be important. ${ }^{15}$ Remember that truth and falsity are properties of propositions (see note 9).

${ }^{16}$ We can of course get the same conclusion using Ryle's 'namely-rider.' Although B\&E think in their book that both the Liar sentence and the Truth-teller sentence express propositions, they do not present any substantial argument for that. 
sentences, have physical appearances, phonetical or visual. As such they are easily identifiable. However, propositions do not seem to have such physically identifiable appearances, which makes their Identification difficult. And the identification of the Liar proposition is virtually impossible because of the kind of vicious circularity explained above. We think of this as showing that there is no proposition like the Liar proposition. If there were such a proposition, we would have to be able to explicitly identify it. We may similarly argue that the Truth-teller sentence "This proposition is true" does not express any proposition; in other words, there is no proposition like the Truth-teller proposition. ${ }^{17}$

In the above we found a vicious circle which makes it impossible for us to find the referent of the phrase 'This proposition' in the Liar sentence. However, if we assume that the Liar sentence expresses a proposition, i.e., the Liar proposition, we will find another vicious circle. Let us assume that the Liar sentence expresses the Liar proposition. Then the Liar proposition has a truth value. Let us try to determine its truth value. What the Liar proposition claims is that it is false. Thus, in order to determine the truth of the claim made by the Llar proposition, we have to examine whether or not the Liar proposition is false; that is, we again have to examine the Liar proposition and determine its truth value. We have come back to the original question. This is a vicious circle which is different from the one explained above. ${ }^{18}$ Furthermore, the principle of bivalence for propositions leads us to the conclusion (contradiction) that the Liar proposition is true if $i t$ is false or that it is both true and false. From these two points, we again have to abandon the assumption that the Liar sentence expresses a proposition.

We did not use the notion of a situation in the above. We can of course relate some situation to the Liar sentence (not to the Liar proposition) by saying, "I now state the Liar sentence "This proposition is false"." Let $s$ be the situation in which I just stated the Liar sentence. Then the statement which I just now made using the Liar sentence is related to 8. But bringing $s$ into the problem of the paradox seems to be of no use because there seems to be no fact of the matter in and around $s$ which can help us determine the truth value of the Liar proposition, if any, expressed by the Liar sentence. For this reason, we do not agree with B\&E that the notion of a situation plays an essential and indispensable role in solving the Liar paradox, although we admit that it plays a very important role in it.

Let us now consider the following pair of sentences in Kripke (1975):

${ }^{17}$ As can be seen below, a strengthened Liar paradox arises here.

${ }^{18}$ Here we have to realize that there is no empirical fact of the matter which can help us determine the truth value of the Liar proposition. 
(a)

(b)

Most of Nixon's assertions about Watergate are false, Everything Jones says about Watergate is true,

where (a) was asserted by Jones and (b) by Nixon. ${ }^{19}$ Suppose 8 is a situation in which (b) is the only assertion by Nixon about Watergate and in which Jones asserted (a) and exactly one other statement about Watergate which is known to be false as a matter of fact. Then (b) is false and therefore (a) is true since (b) is Nixon's sole assertion about Watergate. In this situation s, both (a) and (b) make perfectly good sense or express proposittons and moreover (a) expresses a true proposition and (b) expresses a false proposition. However, there are many other situations in which the two statements do not make sense or do not express propositions. Let $s$ be a situation in which (a) is Jones' sole assertion about Watergate and in which (b) is Nixon's sole assertion about Watergate. In this situation s, (a) and (b) can be identified with (a) and (b), respectively:

(a)

The statement (b) by Nixon is false.

The statement (a) by Jones is true.

Then we see a vicious circle if we assume that both $(a)$ and $(b)$ express propositions in s. Suppose they both express propositions in $s$. Then they have truth values. But the truth value of $(a)$ depends on that of $(b)$, and the truth value of $(b)$ depends on that of $(a){ }^{20}$ There are no facts of the matter in and around $s$ which can help us determine the truth values of $(a)$ and (b). This vicious circle again suggests that the assumption that both (a) and (b) express propositions is wrong. Moreover, the principle of bivalence for propositions leads us to contradictions; if $(a)$ is true then $(b)$ is false and thus $(a)$ is false, and if $(a)$ is false then $(b)$ is true and thus $(a)$ is true. So, $(a)$ is true iff $(a)$ is false. Similarly, $(b)$ is true iff $(b)$ is false. Therefore, by a reductio argument, we have to abandon the assumption that both $(a)$ and

${ }^{19}$ Kripke (1975). Kripke, Saul. "Outline of a Theory of Truth." The Jourmal of Philosophy. Vol. 72 (1975): 690-716. p. 691. B\&E also say that the sentence "This proposition is expressible in English using fewer than twelve words" makes sense and expresses a proposition. But we do not think so. If the phrase 'This proposition' is replaced by 'This sentence,' then the resulting sentence will make sense and express a proposition. Again the problem here is the vicious circularity of the referent of the phrase 'This proposition' in the original sentence.

${ }^{20}$ The phrase 'The statement $(b)^{\prime}$ in (a) refers to (the statement made by the sentence) (b) and similarly the phrase 'The statement $(a)^{\prime}$ in (b) refers to (the statement made by the sentence) (a). So, there seems to be no problem of a vicious circularity of the referent of each of the two phrases. 
(b) express propositions in 5 , because we do not abandon the principle of bivalence for propositions. Thus we claim that neither $(a)$ nor $(b)$ expresses a proposition in s, i.e., that neither (a) nor (b) expresses a proposition in $s^{21}$

The above argument shows that the same sentence may express a proposition in one situation and may not in another. There are of course many such sentences. For example, "The present king of France is crazy" does not express a proposition in any situation in the year 1987. But it expresses a proposition in some situation in the year, say, 1700 and is either true or false in the situation.

In sum, we claim that the Liar sentence does not express any proposition in any situation. This is our solution to the Liar paradox. However, this simple solution needs a bit of complication because it can yield a version of the Strengthened Liar paradox. ${ }^{22}$ We consider this new paradox at the end of this paper.

\subsection{Situations and states of affairs}

B\&E say that an Austinian proposition p consists of two elements. About(p) and Type(p), and that $p$ is the claim that About(p) is of type Type(p). They also say that About(p) is a subset of the class of states of affairs, or facts, and that About(p) is the situation $p$ is about. ${ }^{23}$ However, it is not at all clear which facts should be or should not be included in About(p). Imagine the following situation s:

Max is playing a card game with Claire in his home in Chicago. Max has the three of clubs. A cat named Kay is playing with a toy mouse beside the card players. Max's father is shouting to Max from downstairs that someone is calling him from Boston. Then Max says to Claire, "I don't have the three of clubs." 24

${ }^{21}$ We cannot claim of $(a)$ and $(b)$ that one expresses a proposition but that the other does not. Suppose, e.g., that (a) expresses a proposition but that (b) does not. Then (a) is either true or false. If it is true, then (b) is false; thus it must express a proposition, which is a contradiction. Similarly for the case where $(a)$ is false.

22Professor McCarthy first pointed it out to me with a specific paradoxical sentence which is not exactly the same as the sentence $S_{1}$ in Section 3 . Professor Barwise also pointed it out to me.

${ }^{23}$ For the notions of a state of affairs and of a fact, see note 8.

${ }^{24}$ In this 8, there are many urelements like 'Kay' and 'Boston' which are not included in the Austinian semantics. But it is not essential since we can add those urelements to it. 
Now the proposition $p$ that Max does not have the three of clubs, expressed by Max's statement above, seems to be about s, i.e., About(p)=s. Then the question is which facts 8 contains. It seems clear that 8 contains the fact that Max has the three of clubs. How about the fact that Kay is playing with a toy mouse? Does it also contain the fact that someone, say Tom, is calling Max from Boston? Does it also contain, e.g., the fact that Tom is eating popcorn while calling Max? Where should we stop? Or, are all these facts irrelevant except the one that Max has the three of clubs? Although this last fact is important to the (truth of the) proposition p, other facts in $s$ are equally important as constituents of 8 . If B\&E cannot specify which facts are or are not in $s=A$ bout(p), About(p) does not seem to be well-defined as a set in the universe of AFC/AFA, which seems to damage their account of the Austinian semantics as well as that of the Austinian propositions.

Furthermore, although About(q) for an empirical proposition $q$ like the above proposition $p$ is not so difficult to understand in spite of the vagueness of its contenis which we just discussed above, About(r) for an abstract proposition $r$ is very difficult to understand. If someone states that beauty is the opposite of ugliness, what is the situation that the proposition expressed by this statement is about? How about the situation, if any, that a mathematical proposition like $2+2=4$ is about? Abstract propositions do not seem to be related to any particular or concrete situations. The concept of a situation does not seem to fit abstract propositions or statements. In this sense, the Austinian semantics in which the notion of a situation plays an indispensable role seems to be incapable of handling abstract propositions, or at least needs some modification or extension.

\section{McCarthy's Paradox}

First, we assume that every sentence either expresses a proposition or does not express a proposition, but not both, and that every sentence expressing a proposition, and only such a sentence, is either true or false, but not both. The latter agrees with what we said about sentences in the previous section. ${ }^{25}$ Let us consider this sentence $S_{1}$ :

$S_{1}$ : Either $S_{1}$ expresses a false proposition or $S_{1}$ does not express a proposition. ${ }^{26}$

25see pp. 4-5 of this paper.

${ }^{26}$ That a sentence expresses a proposition depends on situations in which the sentence is used. Thus, strictly speaking, the sentence $S_{1}$ must instead be expressed as: 
Now $S_{1}$ is paradoxical as seen below. This is a strengthened Llar paradox, which we call 'McCarthy's paradox. ${ }^{27}$

(i) $S_{1}$ expresses a prop. $\square S_{1}$ Is true $\triangle S_{1}$ is false.

(or)

$S_{1}$ is false $\square S_{1}$ is true.

(ii) $S_{1}$ does not express a prop. $\square S_{1}$ is true.

What (i) says, e.g., is this: Suppose $S_{1}$ expresses a proposition. Then $S_{1}$ is either true or false. If $S_{1}$ is true, then what $S_{1}$ claims is the case. Since we are assuming that $S_{1}$ expresses a proposition, the second disjunct of $S_{1}$ is not the case. So, the first disjunct of $S_{1}$ must be the case, which means that $S_{1}$ is false. A contradiction. Similarly, if $S_{1}$ is false, then we get a contradiction. Similarly for (ii).

McCarthy's paradox seems to show, as a reductio argument, that at least one of the two assumptions stated at the beginning of this section is wrong. Since we keep the latter assumption in this paper, we take it that the assumption that every sentence either expresses a proposition or does not express a proposition (but, not both) is wrong. In other words, the predicate 'expressing a proposition' is not totally defined, or it is undefined for a sentence like $S_{1}{ }^{28}$ This is our solution to McCarthy's paradox.

$S_{1}$ : Either $S_{1}$ expresses a false proposition in some situation or $S_{1}$ does not express any proposition in any situation.

We, however, do not use the above version of $S_{1}$ here for simplicity's sake. In this section, we do not take situations into consideration.

27 To get McCarthy's paradox, strictly speaking, the two assumptions stated at the beginning of this section are not enough. We also need Tarski's biconditionals and the principle of bivalence for propositions. We also need to assume that every sentence expressing a proposition expresses a unique proposition. We may assume this last assumption because in this section we disregard the notion of a situation when we consider propositions (see note 26 above). Two different situations can make one and the same sentence express two different propositions with possibly two different truth values. However, because of our disregard of situations, one single sentence can express at most one proposition.

${ }^{28}$ In other words, $S_{1}$ cannot be in either the extension or the antiextension of the predicate 'expressing a proposition.' 
Let us call sentences like $S_{1}$ indeterminate ${ }_{1}{ }^{29}$ That is, they are indeterminate as to expressing propositions. Let us now assume that for each sentence $A$, exactly one of the three holds: (1) A expresses a proposition, (2) A does not express a proposition, and (3) A is indeterminate ${ }_{1} .{ }^{30}$ Then we get another paradox, which we call the 'Strengthened McCarthy's paradox,' generated by the following sentence S:

$S_{2}$ : Either $S_{2}$ expresses a false proposition, or $S_{2}$ does not express a proposition, or $S_{2}$ is indeterminate 1 .

If $S_{2}$ expresses a proposition, then it is either true or false; if it is true then it is false by the first disjunct of $S_{2}$ and if it is false then it is true again by the first disjunct of $\mathbf{S}_{2}$. If $\mathbf{S}_{2}$ does not express a proposition, then it expresses a true proposition by the second disjunct of $S_{2}$. If $S_{2}$ is indeterminate 1 , then it again expresses a true proposition by the last digjunct of $S_{2}$. In any case, we get a contradiction. However, this again seems to show the incorrectness of the assumption that for each sentence $A$, exactly one of the three holds: (1) A expresses a proposition, (2) A does not express a proposition, and (3) A is indeterminate1. There is at least one sentence which does not satisfy this assumption. Let us call such a sentence 'indeterminate2.' $S_{2}$ is indeterminate2. We, however, cannot assume that for each sentence $A$, exactly one of the four holds: (1) A expresses a proposition, (2) A does not express a proposition, (3) A is indeterminate 1 , and (4) $A$ is indeterminate. If we assume this, we will get a Strengthened Strengthened McCarthy's paradox.

In general, we may define an infinite sequence of sentences $S_{1}, S_{2}, S_{3}$, ..., $S_{n v}$... as follows:

$S_{1}$ : Either $S_{1}$ expresses a false proposition or $S_{1}$ does not express a proposition.

$S_{2}$ : Either $S_{2}$ expresses a false proposition or $S_{2}$ does not express a proposition, or $S_{2}$ is indeterminate1.

$S_{3}: \quad$ Either $S_{3}$ expresses a false proposition or $S_{3}$ does not express a proposition, or $S_{3}$ is indeterminate ${ }_{1}$ or $S_{3}$ is indeterminate 2 .

${ }^{29}$ The subscript ' 1 ' is attached to make the arguments below uniform.

${ }^{30}$ Besides this assumption, we still hold the second assumption stated at the beginning of this section as well as those in note 27 above. We keep them throughout this section. 
$S_{n}: \quad$ Either $S_{n}$ expresses a false proposition, or $S_{n}$ does not express a proposition, or $S_{n}$ is indeterminate 1 , or ..., or $S_{n}$ is indeterminate $\mathrm{n}_{\mathbf{1}-1}$.

$S_{1}$ can yield McCarthy's paradox. And, for each $n \geq 2, S_{n}$ can yield a strengthened version of McCarthy's paradox if we assume that for each sentence A, exactly one of the $n+1$ conditions holds: (1) A expresses a proposition, (2) A does not express a proposition, (3) A is indeterminate $1, . .$, and $(n+1) A$ is indeterminate $e_{n-1}$. We, however, understand this to show that such assumptions $(n \geq 2)$ are wrong, as we have understood that $S_{1}$ shows that the assumption that every sentence either expresses a proposition or does not (but not both) is wrong. Although this yields an infinite sequence of getting a paradox and solving it, it does not seem unreasonable to regard such an infinite process as a whole as a solution to all such paradoxes. 31

Let us finally remark that in the above, the fancy predicates like

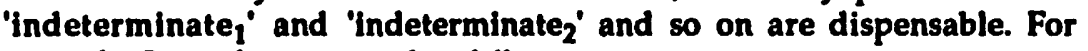
example, $S_{2}$ can be expressed as follows:

$S_{2}$ : Either $S_{2}$ expresses a false proposition, or $S_{2}$ does not express a proposition, or $S_{2}$ has neither the property 'expressing a proposition' nor the property 'not expressing a proposition.'

This version of $S_{2}$ can produce a version of the Strengthened McCarthy's paradox if we assume that for each sentence $A$, exactly one of the three holds: (1) A expresses a proposition, (2) A does not express a proposition, (3) A has neither the property 'expressing a proposition' nor the property 'not expressing a proposition.' This assumption is essentially the same as the one given when we discussed the Srengthened McCarthy's paradox. As we denied the latter assumption there, we deny the former here. The point is that not the predicates 'indeterminate' (iz1) but the above kinds

${ }^{31}$ Our solution somewhat reminds us of Tarski's hierarchy of infinitely many languages in which he solved the Llar paradox(es). Also our solution has a good connection with McCarthy (1985). McCarthy, Timothy. "Abstraction and Definability in Semantically Closed Structures." Joumal of Philosophical Logic. Vol. 14 (1985): 255-266. 
of assumptions are essential to producing and solving strengthened McCarthy's paradoxes. ${ }^{32}$

\section{A Summary}

The most important difference between our position and B\&E's is that we do not think Liar-like sentences, l.e., sentences involving vicious circularity, express propositions, while they do. It is our belief that our solution to the Liar paradox, l.e., regarding the Liar sentence as expressing no proposition in any situation, is more natural and reasonable than B\&E'8. However, it is one of their great contributions to the problem of the Liar paradox that they greatly clarifled, using the concept of situation, the meanings of sentences involving the notion of truth. Their work has set up a new stage of the study of the Liar paradox.

${ }^{32}$ When we consider strengthened McCarthy's paradoxes in classical formal languages, primitive (not definitional) predicate symbols corresponding to 'indeterminate' (i>1) seem to be needed. 